\title{
Ethical Dilemmas in Euthanasia of Small Companion Animals
}

\author{
Marcela Rebuelto*
}

Pharmacology, Veterinary Sciences Faculty, University of Buenos Aires, Argentina

\begin{abstract}
Animal ethics is a complex subject, and only recently the concern about the moral status and rights of non human animals, is subject of debate. In this paper I will deal mostly with the ethics in deciding euthanasia in the small animal clinical setting. My purpose is to lay out some issues about animal euthanasia, with the hope of helping veterinarians to analyze the relevant ethical concerns, as this is a field of the veterinary practice in which there are many conflicts for taking the right decision and justifying the election. Euthanasia of small companion animals is a highly stressful situation for veterinarians and may be source of ethical dilemmas. The final choice must prioritize the benefit for the patient while respecting the owner's freedom of choice.
\end{abstract}

\section{INTRODUCTION}

During the Nuremberg trial, after the ending of World War II, the scientific (and the entire) world was horrified by the atrocities that in the name of scientific research the nazi regime had done to human beings. The Nuremberg Code, a result of these revelations, stated unequivocally the principle of voluntary consent of human beings prior to enrolling in any research. From then on, the protection of the welfare of human beings participating in scientific research was subject of debate. The Belmont report, the Declaration of Helsinki and amendments, developed by the World Medical Association, and the International Ethical Guidelines for Biomedical Research Involving Human Subjects, developed by the Council for International Organizations of Medical Sciences (CIOMS) stated several issues of biomedical research ethics, referring to the basic ethical principles of respect for persons, beneficence, non maleficence, and justice that should assist in resolving the ethical problems involved in the conduct of research with human subjects.

Meanwhile, significant steps were taken in the field of animal ethics. In 1959 two English scientists, zoologist and psychologist William Russell, and microbiologist Rex Burch, carried out a thorough study of the ethical aspects and "the development and progress of humane techniques in the laboratory". These scientists formulated the Three R's approach of replacement (use of nonsentient organisms rather than higher animals for experiments), reduction (use of the smallest possible number of animals that warrants obtaining the best quality and most precise information), and refinement (use of procedures that reduce the incidence or severity of pain and/or distress) in research involving laboratory animals in their book "The Principles of Humane Experimental Technique". In 1962, Ruth Harrison draw the attention of scientists and public in general to the lack of welfare considerations, mostly space restrictions, on the animal husbandry practices on veal calves, poultry and swine production, with her book "Animal Machines". As a consequence, in 1965 the

*Address correspondence to this author at the Pharmacology, Veterinary Sciences Faculty, University of Buenos Aires, Chorroarín 2801427 Buenos Aires, Argentina; E-mail: rebuelto@fvet.uba.ar
Brambell report outlining basic ethical and biological principles for animal husbandry, was published in Great Britain summarizing the deliberations of the specially appointed Brambell Committee. In 1975, Australian philosopher Peter Singer publishes his book "Animal Liberation", considered as one of the most important works in animal rights and moral equality for animals, describing the cruelty involved in the common practices of laboratory testing on animals. Even though both the Declaration of Helsinki and the International Ethical Guidelines for Biomedical Research Involving $\mathrm{Hu}$ man Subjects-CIOMS state that medical research involving human subjects must be based when appropriate, on previous animal experimentation, only the paragraph 11 of the Declaration of Helsinki states that "the welfare of animals used for research must be respected".

\section{EUTHANASIA IN VETERINARY MEDICINE}

Veterinarians may deal with various circumstances in which death of the animal is required, as in research, food production, during the outbreak of a disease, and at the clinical setting. These circumstances, even though all of them implying the death of animals, are quite different.

Occasionally, the research experience includes the death of the subjects, usually laboratory animals such as rats, rabbits, mice or guinea pigs. Even though these animals are considered sentient organisms, this is, they experience pain and pleasure, each individual of this group is not significantly different from another, and there are considered as an "experimentation subject" by researchers, an object of scientific inquiries with none emotional attachment at all. In a similar way, food animals may be considered merely as "units of production", and its value to owners and attending veterinarians is primarily economic. Killing in the food or fur productions, in the outbreak of diseases (termed culling), and in research usually implies the sacrifice of large number of animals. This is a difficult task, and several methods as the administration of a gas mixtures using carbon dioxide, oxygen, argon o nitrogen or other inert gas, cervical dislocation, decapitation and electrical procedures have been reported [1]. The standard recommended methods for euthanasia, as the administration of lethal injections, or the administration of an anesthetic in the animal's food or water have, 
indeed, many limitations, are time consuming and produce dubious and irregular results when large numbers of individuals, as cattle or poultry or laboratory animals, are involved. But even though those procedures are not feasible in such circumstances, some issues must be addressed for developing specific protocols for killing large numbers of animals, such as the protection for the health and safety of the personnel when handling the animals or applying the killing procedures. Ethics plays a major role in the concern on the welfare of the animals, and any method should provide a rapid loss of consciousness and produce a painless, quick death. Animals intended for food should be slaughtered humanely, and performed as required by the local regulations, usually stunning of the animal followed by exsanguination. Some religions, i.e. Jewish, may need special procedures, as kosher slaughter, however they must always respect the welfare of the animals. Massive killing during certain diseases outbreaks in farm animals such as poultry or cattle may be required as a disease control measure adopted in order to minimize the risk of disease spreading to other animals or farms, or even to avoid the risk of the disease transmission to human beings. A recent example of such a need has been seen with the H5N1 virus avian influenza outbreak started in 2003 and still ongoing, during which the WHO decided that a standard control measure was to kill flocks of poultry that were infected or may had been exposed to avian flu, as this strain of avian influenza is highly pathogenic and potentially zoonotic. The 2001 foot and mouth disease epidemic, on the other hand, led Great Britain to the slaughter of more than six million animals, in order to avoid possible transmission to other animals and quick spreading of the disease that would produce high economic loses.

Many guidelines and recommendations that are beyond the scope of this paper, have been developed dealing with the appropriate and humane procedures for killing animals during research, food production and disease outbreaks. In this paper I will deal mostly with the ethics in deciding euthanasia in the small animal clinical setting. My purpose is to lay out some issues about animal euthanasia, with the hope of helping veterinarians to analyze the relevant ethical concerns, as this is a field of the veterinary practice in which there are many conflicts for taking the right decision and justifying the election. Animal ethics is a complex subject [2], and only recently the concern about the moral status and rights of non human animals is subject of debate. Veterinarians are quite unaccustomed to making ethical reflections, however, they are strongly concerned with the ethical aspects about decisions related to the prescribing and proceedings of euthanasia in a rational, respectful and responsible way.

\section{EUTHANASIA IN SMALL COMPANION ANIMALS}

The term euthanasia is derived from the Greek ( $e u$ meaning "good", and thanatos meaning "death"). For the Panel of Euthanasia appointed by the American Veterinary Medical Association [1], euthanasia is "the act of inducing humane death in an animal". In the clinical veterinary practice, euthanasia is regarded as an ethical procedure when the veterinarian considers inhumane to prolong the extremely painful or poor quality life of a patient that cannot be relieved by treatment. The difference between these two definitions is extremely important, and may be source of ethical conflicts when deciding euthanasia of a companion animal [3]. Ethical concerns rise because, for a veterinarian dealing with pets, they not only deserve considerations as sentient individuals, but also its interaction with humans must be taken into account. The relations of the human beings with pets are quite different from those of a scientist with the laboratory animal, or those of a veterinarian with cattle or poultry. Pets may be central to the feelings of care and love of a family, and owners may feel strongly his or her pet is a very much loved companion, and consider they are responsible for their life and well being.

Euthanasia of small animals, as cats and dogs, is a highly stressful situation not only for the owner of the animal to which euthanasia will be done, but also to those who have responsibilities in performing such procedure, as veterinarians or workers on animal shelters. Owners most common response to euthanasia is grief at the loss of his or her pet and guilt about their consent to euthanize their pet [4]. As a prove of how distressing this situation is for the owner o client, in dogs, cats and horses or other animals regarded as pets, euphemisms such as "put to sleep" or "put down" are used for euthanasia. Veterinarians are also affected negatively by euthanasia, as the animal has usually been his or her patient for long, and they have become affectionately attached to it, or because they sympathize with the owner, and cope with different ways to the euthanasia of their patients [5]. Veterinarians feel strongly about euthanasia, and the correspondence published by The Veterinary Record as "Views on Euthanasia" during July 2007 shows the diversity of views veterinarians have on such topic. Opinions justifying euthanasia when the animal is "no longer wanted for its previously intended use" [6], to the defense of the inherent animal value that exists by virtue of "their being sentient individuals with sensations, and social and emotional lives" [7], were published, showing the extent of the debate. Employees providing daily care and attention to the homeless and unwanted dogs and cats with euthanasia responsibilities are also emotionally affected, showing high turnover rates positively correlated to euthanasia rates [8].

As deciding on euthanasia is a hard choice for an owner to make, veterinarians may face the fact that clients rely heavily on the veterinarian's judgment, not only because of the Aesculapian authority acknowledged by the owner, this is, the authority that accompanies the knowledge to heal [9], but also for avoiding the feeling of responsibility, or guilt of thinking that they are abandoning or letting his or her pet down. Many owners request euthanasia because they realize that his or her animal suffers a serious disease that cannot be successfully treated and compromises the quality of the animal's life. On the contrary, many owners refuse euthanasia for his or her pets, even though the animal is suffering, and the veterinarian may have to explain to the distressed owner, in an open and honest way, why euthanasia is in the best interest of his or her pet. Quite different, however, is when an owner of a perfectly healthy pet asks it to be euthanized, because of treatable sickness or behavioral problems (inappropriate elimination, aggressive behaviors, excessive barking), or because social reasons (being no longer useful, moving to a smaller home) [10]. In these circumstances, some veterinarians consider a moral dilemma how to comply with the client's right to make decisions regarding his or her pet 
versus the responsibility the veterinarian has to alleviate the suffering of his patients and provide and ensure them humane and scientific care. These are difficult situations in clinical practice, and probably one of the most stressful for a veterinarian. American bioethicist and philosopher Bernard Rollin states that the fundamental question of veterinary ethics is: To whom does the veterinarian owe primary obligation: animal or owner? [11]. In the topic of euthanasia, the potential ethical dilemmas are: Do I respect a client's wish not to euthanize a suffering animal?, or: Do I euthanize a healthy dog or cat? Let's analyze the firs one, the owner with no wish to euthanize his animal. Even though the veterinarian is the one with the scientific knowledge to decide when euthanasia is acceptable, client consensus is an inescapable requirement. If a veterinarian proceeds to euthanize a pet without the owner's consent, this procedure is ethically wrong, whatever the animal health state may be. Thus, in this case, the veterinarian must try to demonstrate the owner why his or her pet's quality of life is no longer a good one, and why there are no treatments that can change this circumstance. What about the second dilemma, that one about euthanizing a healthy dog for convenience reasons? In many countries, the owner has the right of the ownership, the animal belongs to him. This allows an owner to request, legally, the euthanasia for his pet, and some clients believe that veterinarians have to follow their wishes and convenience blindly, with no regard for the animal, acting according to the "garage mechanic" model instead of the "pediatrician" model [9] as they are the paying customers. However, veterinarians have professional obligations towards their patients, and if the owner wants to sacrifice his or her animal, the veterinarian must decide if it is ethically acceptable, and by no means must be coerced to proceeding to euthanasia. To accede to the client's request for euthanasia, a veterinarian must believe that it is justified because it is in the best interest of his patient, and because it is the appropriate solution to the patient's problems. Moreover, to accede to a client's request of euthanizing his or her pet, and failing to do so (i.e., rehoming the animal) is unethical even if it was done because of the veterinarian's moral objections.

Decisions related to euthanasia deserve ethical considerations, and even more in such conflicting circumstances as outlined above. As it is well known, when making ethical judgments, the principles of beneficence, non maleficence, respect for persons and justice are applied equally but not absolutely to medical practice, public health and research in human beings. For euthanasia decision-making, two principles, autonomy and beneficence, are the most relevant [12].

Respect for persons means that individuals should be treated as autonomous agents, this is, as individuals capable of deliberation about personal goals and of acting under the direction of such deliberation. In human medicine, a patient is autonomous if he or she can decide in consenting to or refusing a treatment using certain decision making capacities, this is, if he or she has the ability to understand and prioritize the convenience of the treatment, the potential drawbacks, other treatment options, and decide whether this or that treatment is convenient for him or her. Thus defined autonomy, animals are definitely not autonomous. If we define autonomy as the capacity of govern oneself, however, animals are autonomous, as they can live happily and successfully without human beings. However, in veterinary medicine, as it is impossible to communicate with the patients, this is, the animals, when deciding on medical or surgical treatments, as in deciding euthanasia, the owner's autonomy must be respected.

The principle of beneficence is understood as having a duty to act in the best interest of the patient, and is closely related to the quality of life of the patient. In euthanasia, both principles , beneficence (do the good) and non maleficence (do no harm) are opposite, as nobody can think taking an animal's life is "doing no harm". In euthanasia, the principle of non maleficence must be outweighed by the principle of beneficence. As opposite to the principle of autonomy, related to the owner, the principle of beneficence applies to the animal, and the well being (or quality of life) of the patient is central in the decision-making process.

Quality of life is both difficult to define and to assess. In human medicine, it is very difficult to assess a patient's quality of life, as this is a subjective feeling, and varies with identical situations in different human beings. In veterinary medicine, the veterinarian is qualified to decide, quite accurately, if the animal is suffering unbearably and hopelessly, and if life quality is unlikely to improve, whereas the owners, as they know their pet so well, can assess by observation of changes in the animals behavior, if the animal is suffering $[13,14]$. In animals, McMillan proposed that quality of life is a multidimensional array of affective states, and that it refers to a state of mind resulting from the balance between comfort-discomfort and pleasant-unpleasant feelings. Subjective feelings are central and exclusive in all interpretations of quality of life in animals. Needs, social relationships, stress, and health are important factors contributing to quality of life [15]. Even in animals, the experience of pain (emotional and physical) and suffering will always be a subjective experience that affects negatively their's quality of life $[16,17,18]$. To the animal mind, life is pleasant or unpleasant depending on if the are with pain or not, with hunger or not, with thirst or not, etc. It is quite probable that, to animals, death holds no singular importance. A very sick or painful animal is incapable of hoping to get cured, or for the pain to stop, thus, prolongation of life at any cost to its quality is not justified.

When proceeding to the euthanasia of a pet, the following issues must be addressed:

i) Decision making process must take into account and balance factors as age of the animal, possible treatments, degree of injuries or disease, quality of life and welfare of the animal.

ii) The circumstances and consequences of the concrete facts in each particular case must be analyzed thoroughly, considering both the best for the patient and the best for the owner. Each case is unique.

iii) The overwhelming moral imperative of a vet is the welfare of its patients, this eventually may be opposed to the convenience of his clients (as Bernard Rollin [9] states, the pediatrician versus the garage mechanic model). Thus, by no means a veterinarian must be coerced into proceeding to euthanasia of a healthy animal on basis of social or convenience reasons even though pets may be considered as legal properties of their owner. 
iv) Euthanasia is best performed within a strong clientveterinary relationship, this is, not to be done by a referred veterinarian, unless there is an emergency case. This is because I consider that is a difficult step for an affectionate owner to give, albeit an appropriate one, and his or her concerns and wishes must be given due consideration by a professional that is familiar both with client and patient. However, I acknowledge the convenience of the specialist deciding euthanasia in those cases in which knowing the client makes extremely difficult for a veterinarian to propose such procedure, provided that the specialist has stayed in good communication with the referring clinician [14].

v) Procedures must be carried out avoiding all pain and distress as possible, with the highest sense of responsibility, respect for life and compassion. Loss of consciousness must be reached rapidly. Some authors, as Temple Gradin, a well known American humane livestock facility designer, have proposed the use of sacred rituals when performing euthanasia [5]. Handling previous to euthanasia must be proper and gentle.

vi) It must be carried out by a veterinarian acting in his or her professional capacity. In some countries, i.e. Italy, the law orders that euthanasia must be carried out by a veterinarian [19].

vii) If the owner chooses to be present, emotional effects that result of observing the procedure must be taken into account when deciding which method use, and the veterinarian will benefit if he explains carefully what drug will be administered to the pet, why it was selected, and how and when the animal will respond. The procedure must allow the owner to remain, talk and touch his or her pet as long as possible, and must be done carefully, looking for minimizing the distress of the grieving owner, and respecting his or her need of privacy [20]. There are some effects especially disturbing to owners, such as vocalization or convulsions. Esthetical requirements, however, must never outweigh the primary ethical requirements of a rapid, painless and distress-free death. The use of neuromuscular agents, such as curare-like drugs, is unacceptable for euthanasia.

\section{DECIDING ON EUTHANASIA}

I have found helpful when facing the difficulties of deciding whether or not euthanize an animal, to develop the reasoning as proposed by Bebeau and Pimple [21].

I make an interpretation of the situation identifying what actions are possible (euthanasia/no euthanasia/other alternatives), who would be affected by each course of actions (the owner, other family members, myself), and how these interested parties would regard such effects on the animal's benefit.

First, I identify the ethically relevant components of the situation, i.e., prognosis of the injury or disease, quality of life of the animals (the owner's own assessment is a very important factor), relationship (feeling) owner/patient and other members of family/patient, financial restriction that risks treatment, life expectancy of the patient, alternatives to other (maybe less efficacious) treatments, treatment considered as the gold standard for the situation.
Second, I define if there are other morally justifiable alternative courses of action than euthanasia, as rehoming of the pet or palliative cares.

Third, I identify my personal values in this situation, and make a judgment about which course of action I think is the "right" solution to the problem.

Fourth, I identify the owner's personal values in this situation, and the reasons why the owner wants/does not want to follow the proposed action.

Ideally, veterinarian and pet owner should agree on the decision. If they do not, two possible ethical dilemmas rise, as the obligation of the veterinarian to act in the benefit of his patients (principle of beneficence) conflicts with the right of the owner to decide on his or her pet (principle of autonomy). On the one hand, the veterinarian feels euthanasia is the right course of action and the owner does not, on the other hand, the veterinarian feels the request of euthanasia of an owner is not justified. In these situations I propose referring the patient to a specialist, as the specialist's distance from the client may enhance the influence of the Aesculapian authority, and may help the owner to decide on the right course of action [14]. If the veterinarian thinks euthanasia is premature, or unjustified, he or she must discourage such procedure, and must not feel compelled to validate such procedure.

In all instances, take your time before reaching a decision. As Morgan and McDonald [22] rightly stated, "hasty decisions in morally charged situations could have long-term consequences for patients, clients, veterinarian and the profession". The final choice must prioritize the benefit for the patient while respecting the owner's freedom of choice. But above all, the veterinarian must be certain that in such situation to have the animal euthanized is morally justified. If the veterinarian is not sure of the rightness of the procedure, even though the owner, in his or her legal right, requires euthanasia, it is my view that the veterinarian must not comply. Euthanasia is a distressing enough experience in a clinical setting to add guilt and regret to the process.

\section{REFERENCES}

[1] AVMA Guidelines on Euthanasia. Downloaded from http://www. avma.org/issues/animal_welfare/euthanasia.pdf, date of access: August 2008. 2007.

[2] Fox MW. Principles of veterinary bioethics. J Am Vet Med Assoc 2006; 229: 666-7.

[3] McMillan FD. Rethinking euthanasia: death as an unintentional outcome. J Am Vet Med Assoc 2001; 219(9): 1204-6.

[4] Adams CL, Bonnet BN, Meek AH. Predictors of owner response to companion animal death in 177 clients from 14 practices in Ontario. J Am Vet Med Assoc 2000; 217(9): 1303-9.

[5] Manette CS. A reflection on the ways veterinarians cope with the death, euthanasia, and slaughter of animals. J Am Vet Med Assoc 2004; 225(1): 34-8.

[6] Allen T. Views on euthanasia. Vet Rec 2007; 171: 72.

[7] Gardiner A. Views on euthanasia. Vet Rec 2007 161: 143-144.

[8] Rogelberg SG, Reeve CL, Spitzmüller C, et al. Impact of euthanasia rates, euthanasia practices, and human resource practices on employee turnover in animal shelters. J Am Vet Med Assoc 2007; 230(5): 713-9.

[9] Rollin BE. The use and abuse of Aesculapian authority in veterinary medicine. J Am Vet Med Assoc 2002; 220:1144-9.

[10] Scarlett JM, Salman MD, New JG, Kass PH. The role of veterinary practitioners in reducing dog and cat relinquishments and euthanasias. J Am Vet Med Assoc 2002; 220(3): 306-11. 
[11] Rollin BE. Euthanasia and quality of life. J Am Vet Med Assoc 2006; 228(7): 1014-6.

[12] de Haan J. The ethics of euthanasia: advocates' perspectives. Bioethics 2002; 16(2): 154-72.

[13] Wojcjechowska JI, Hewson CJ. Quality-of-life assessment in pet dogs. J Am Vet Med Assoc 2005; 226 (5): 722-8.

[14] Rollin B. The ethics of referral. Can Vet J 2006; 47: 717-8.

[15] McMillan FD. Quality of life in animals. J Am Vet Med Assoc 2000; 216(12): 1904-10.

[16] Hellyer PW. Treatment of pain in dogs and cats. J Am Vet Med Assoc 2002; 221(2): 212-5.

[17] McMillan FD, Rollin B. The presence of mind: on reunifying the animal mind and body. J Am Vet Med Assoc 2001; 218: 1723-7.
[18] McMillan FD. A world of hurts-is pain special? J Am Vet Med Assoc 2003; 223(2): 183-6.

[19] Passantino A, Fenga C, Morciano C, et al. Euthanasia of companion animals: a legal and ethical analysis. Ann Ist Super Sanitá 2006; 42(4): 491-5.

[20] Martin F, Ruby KL, Deking TM, Taunton AE. Factors associated with client, staff, and student satisfaction regarding small animal euthanasia procedures at a veterinary teaching hospital. J Am Vet Med Assoc 2004; 224(11): 1774-9.

[21] Bebeau MJ, Pimple KD. Moral Reasoning in Scientific Research. Indiana University, downloaded of http://poynter.indiana. edu/, date of access: January 2008: 1995.

[22] Morgan CA, McDonald M. Ethical dilemmas in veterinary medicine. Vet Clin North Am Small Anim Pract 2007; 37(1):165-79.

(C) Marcela Rebuelto; Licensee Bentham Open.

This is an open access article licensed under the terms of the Creative Commons Attribution Non-Commercial License (http://creativecommons.org/licenses/by$\mathrm{nc} / 3.0 /$ ) which permits unrestricted, non-commercial use, distribution and reproduction in any medium, provided the work is properly cited. 